\title{
Agents for Smart Power Grids
}

\author{
Salem Al-Agtash ${ }^{1,2}$, Hossam Abdel Hafez ${ }^{1}$ \\ ${ }^{1}$ Department of Computer Engineering, German Jordanian University, Amman, Jordan \\ ${ }^{2}$ Department of Computer Science and Engineering, Santa Clara University, Santa Clara, CA, USA \\ Email: salagtash@scu.edu
}

How to cite this paper: Al-Agtash, S. and Hafez, H.A. (2020) Agents for Smart Power Grids. Energy and Power Engineering, 12, 477-489.

https://doi.org/10.4236/epe.2020.128029

Received: July 5, 2020

Accepted: August 10, 2020

Published: August 13, 2020

Copyright (C) 2020 by author(s) and Scientific Research Publishing Inc. This work is licensed under the Creative Commons Attribution International License (CC BY 4.0).

http://creativecommons.org/licenses/by/4.0/

\section{(c) (i) Open Access}

\begin{abstract}
The future of electricity systems will compose of small-scale generation and distribution where end-users will be active participants with localized energy management systems that are able to interact on a free energy market. Software agents will most likely control power assets and interact together to decide the best and safest configuration of the power grid system. This paper presents a design of agents that can be deployed in real-time with capabilities that include optimization of resources, intensive computation, and appropriate decision-making. Jordan 51-bus system has been used for simulation with a total generation capacity of $4050 \mathrm{MW}$ of which $230 \mathrm{MW}$ represents renewable energy. The economic analyses demonstrated the use of smart grid technologies with 2016 generation-load profiles for nominal liquified gas (NLG) prices and $\pm 20 \%$ sensitivity analysis. The results have shown variations in the range of $1 \%$ in the price of $\mathrm{MWh}$ with smart grid technologies. These variations are mainly driven by the fact that agents shift power generation to renewable power plants to produce maximum power at peak hours. As a result, there is a positive economic impact in both NLG $\pm 20 \%$ sensitivity analysis, due to the fact that agents coordinate to better displace expensive thermal generation with renewable generation. It is evident that renewable resources compensate for power at peak times and provide economic benefits and savings.
\end{abstract}

\section{Keywords}

Agents, Electric Power System, Smart Power Grid

\section{Introduction}

The development of smart grids has been progressing rapidly in recent years. Traditional electric power grids began to adopt smart grid technologies [1] [2]. Agents have demonstrated their applicability as innovative tools to manage and 
operate pervasive elements of smart grids. They act as smart self-organizing hardware and software structures in an integrated power system environment that is comprised of generators, distribution substations, transformers, and transmission lines [3]. Intuitively, a smart grid is considered to evolve with grid reconstructive technologies and tools to dynamically optimize grid operations and resources and to incorporate end-user demand response participations [4]. Multiagent systems (MAS) may be designed to inherit SCADA (supervisory control and data acquisition) architecture while accommodating autonomous and intelligence attributes. MAS technologies have been used in diverse power system applications like disturbance diagnosis, restoration, secondary voltage control, and visualization [5] [6] [7]. Their wider implementation has been demonstrated in solar power generation batteries, controllable loads, converters and inverters, demand-side management, data acquisition and grid planning [5] [8]. Agents are implemented as software units which accept signals and interactively prioritize loads, specify time and control status of loads [9].

The specific agent-based solutions for smart grids that are composed of various passive and active system modules have been presented [10]. Decentralized grid arrangements are demonstrated with generation patterns and demand configurations modeled as self-healing agents characterizing transmission, transformers, generation plants, and end-user metering devices. Smart capabilities for visualization, smart sensing automation, and self-healing digitized elements represent the main attributes [11] [12]. The complications of reliability and reassurance, which arise in smart grid, measure agent actions, security and dependability in a multifaceted and vibrant environment. It has been concluded that MAS inherits profits of tractability and extensibility and employs autonomous resolutions like smooth conversion of a grid-networked system to island mode, load shedding, fortifying loads termed as critical, and much more [6]. The aggregate composition of MAS in grid system implementation that considers renewable resources poses a new challenge.

In this paper, we investigate MAS as an appropriate technology for smart grids, while investigating distributed elements necessitating self-sufficiency in their operation and interaction. A smart grid model is presented as a mesh network of micro-grids, which are composed of diverse distributed energy resources with self-possessed smart ICT sub-systems [13]. The Java Agent Development (JADE) Framework is used for agent implementation representing generation, transmission, and distribution elements [14]. The Jordan's 52-bus electric power system is used for illustration. The potential solar and wind energy sources contribute to significant encounters to reduce imports of fuel and preserve environmental and climatic assets. These demonstrations provide in-depth understanding of MAS strategy and development perspectives in the smart grid context with distributed energy resources. Control processes for handling partial distribution from distributed energy resources are used to protect critical loads throughout crises 
while still assisting prioritized non-critical loads belonging to numerous consumers.

The remaining sections of this paper are organized as follows: Section II presents emerging technologies of smart micro-grid systems and integration in agent automation. The design and deployment architecture of agents are given in Section III. An illustrative example and implementation are presented in Section IV. Finally, the paper is concluding in Section V.

\section{Smart Grid}

Power grids are empowered by smart grid technologies and distributed generation, including photovoltaic and wind energy resources [7] [15]. It has been noted that smart grids automate generation, transmission, and distribution to guarantee stable stream of power with minimum blackouts [9]. Smart grids provide efficient tools for power grid control in real-time while maintaining secure and cost-effective operation [16]. Figure 1 shows a model agent-based smart grid. Consumers actively participate in the generation market. They generate power from renewable resources and interconnect with the power grid either for feeding or consuming power. Ultimately, transmission, distribution, and end users are integrated so that power flow is controlled and that environmental concerns are preserved.

\subsection{Intelligent Agents}

Agents represent elements of power system and use standard plug-in interfaces to interact with the energy management system. The system consists of scheduling algorithms, power flow optimization, day-ahead load prediction and generation planning, monitoring and decision making, and supervisory control. Local and remote databases are maintained for power generation, transmission, and distribution. The design of intelligent distribution and operation of agents is tractable and would enable a high-level degree of automation in the power industry. Consumer agent collects information on carbon emissions and energy use from smart meters. It supports the use of energy storage devices as essential ways to decrease peak demand and facilitate integration of intermittent renewable resources. The monitoring and control system manage and operate smart grid

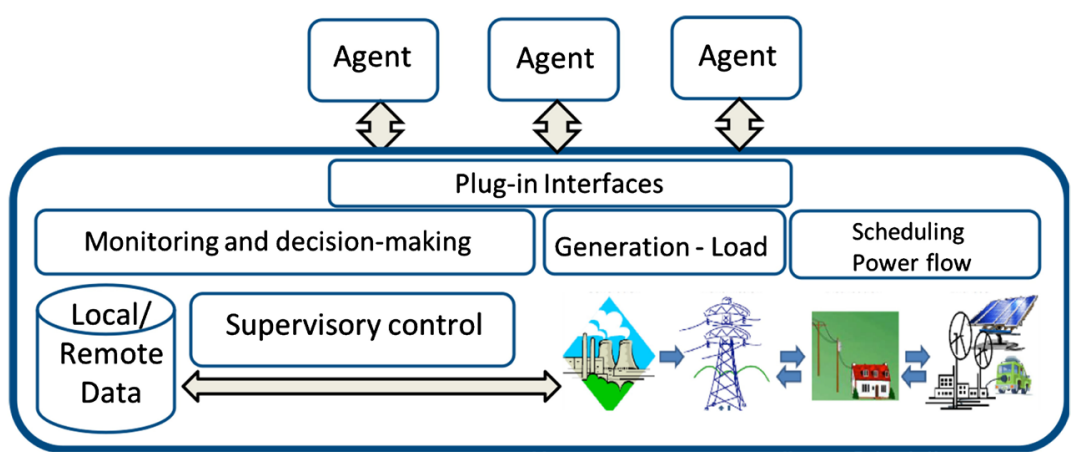

Figure 1. Smart grid proposition. 
system [17]. It integrates various tools that are used to identify instabilities and to integrate renewable energy resources.

\subsection{Smart Technologies}

Intelligent agents may ember smart technologies for automatic generation control, static Var compensators, high voltage direct current control, energy management systems, demand side response, and energy storage. Integration of information and communication technologies (ICT) is essential for real time operation, monitoring, and control of the electric power system and its integration with variety end-user devices and appliances. Users receive real-time price information on cost and carbon intensity. This information is used to forecast forthcoming power demand and supply. Smart meters represent the core components for automating energy use within homes and offices. They are used to manage two-way power flow, where end users reduce energy waste and gain good control over how they consume power [7]. Smart meters interact with residential demand management, self-healing and outage management, and electric vehicle charging. They communicate with load control devices and data repositories using secure communication on the Internet. They integrate into existing utility operations and asset management processes and may reduce grid vulnerabilities and enhance energy efficiency.

\subsection{Power Flow}

The power flow on transmission lines is managed by control centers and sub-stations. Intelligent agents are potential technologies to empower features similar to analytical proficiencies for analysis and monitoring at control centers. A variety of devices are used. The devices of the flexible alternating current transmission system (FACTS), for instance, are generally used to enhance controllability and increase power transfer capability. The high-voltage direct current (HVDC) is used to supply power over extensive distances at minimum losses. It also enables connection between asynchronous grids [17]. The supervisory control and data acquisition (SCADA) system provides analysis of situations and information in real time. Dynamic line ratings deliver a way of classifying existing transporting ability of a unit of network in real-time scenario. Demand response includes various actions that are taken in case of risks due to load shortage or excess. Such contingencies can make demand and supply unbalanced. One of the primary actions is to minimize the usage of electricity when prices are high by shifting load shifting. Agents operate smart power transmission and coordinate with control centers to enhance power utilization, security, and quality and reliability.

\section{Agent Implementation}

Agents are implemented with functionalities that include auto recovery, immune to cyber-attacks, cooperation and coordination with generation mix, and monitoring 


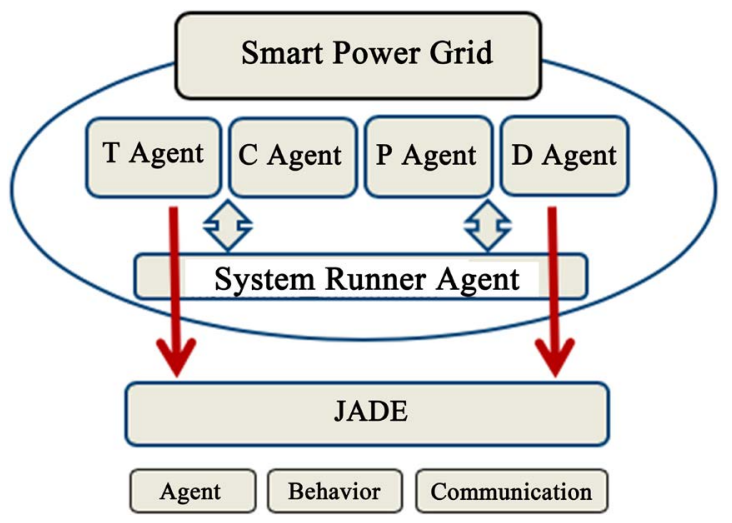

Figure 2. JADE agents component architecture.

and control in real-time. Complex interaction patterns like cooperation, coordination, and negotiation are embedded [13]. We use JADE platform as a generic robust agent architecture to design and implement generation, transmission, and distribution agents of the smart grid [14] [18] [19]. It allows distributed intelligence and simplifies the construction of interoperable agents. The main features of JADE include standardization, portability, dynamism, and versatility. Readymade functionalities and interfaces are provided for custom and application-dependent tasks. JADE is fully compatible with FIPA standards and enables agent platforms to be distributed across different machines, and supports portability [13].

\subsection{Agent Design}

Each agent embeds operational intelligence, decision making, and interactive communication. Its state attributes are common to all agents, including identity, owner, agent type, license, and authorization. An agent reacts to a spectrum of events using embedded behavioral functions and coordinates with its peer agents to meet operational requirements, and to maintain security and reliability. Figure 2 presents JADE agent design with components that include transmission agent ( $\mathrm{T}$ Agent), customer agent (C Agent), generation agent (P Agent), and distribution agent (D Agent). All agents start and create their operational logic defined in SystemRunnerAgent. The JADE library contains all subcomponents necessary for the simulation process, including agent, behavior and communication. The packages "Agent" and "behavior" are used for the purpose of enabling interaction between various JADE agents. The data package carries classes that help in serializing the payload messages from agents. Properties and entities are stored in the Classes from the "model" package. The Java swing libraries and classes contained in the "monitoring" package are used for the implementation of application's front-end. Snippet of an Agent code is given as follows:

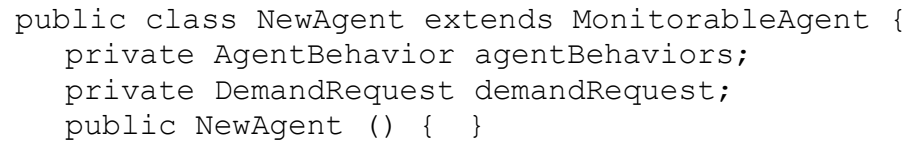




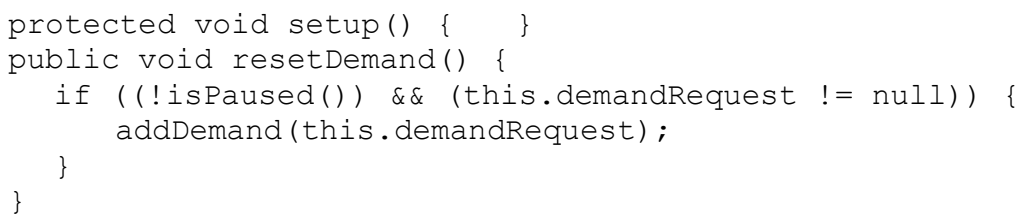

The primitive or composite behaviors are extended by typical Behavior code, defined as follows:

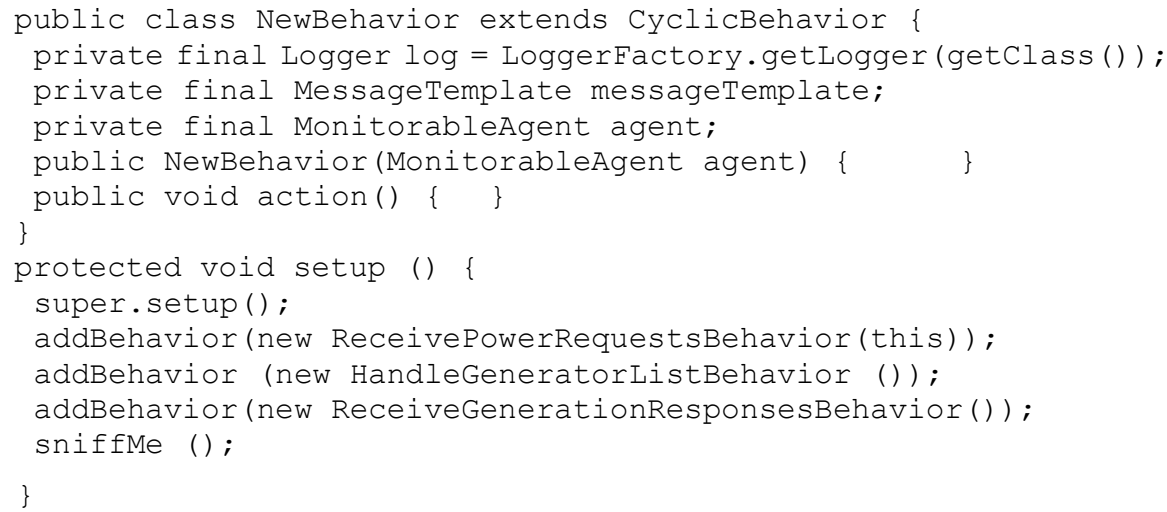

\subsection{Agent Behavior}

Agents perform their tasks independently and operate autonomously [19]. Agent behavior as defined by JADE implements a series of concurrent threads. Whenever a new message arrives, the agent invokes the relevant behavior object [13], eventually derived from one of the following behavior classes:

- CyclicBehavior: handles messages.

- OneShotBehavior: performs only one task.

- WakerBehavior: performs a task after a predefined time.

- TickerBehavior: performs continuously and repetitively.

- ReceiverBehavior: responses to an action.

- Composite Behaviors: combines composite tasks.

- ParallelBehavior: performs tasks simultaneously.

- SequentialBehavior: performs tasks sequentially.

The specific behavior of an agent is mainly driven by the operational characteristics of the underlying device or entity. In studying the Jordan electric power grid, agents are designed to represent generating units, distribution substations, end user customers, and transmission system.

A generation agent $i$ represents the $i^{\text {th }}$ generating unit that injects electric power $g_{i}$ through its $k^{t h}$ connected generation bus $b_{k}$ to the transmission network. It operates under a number of constraints, formally given by $h\left(g_{i}\right)$. The power generation at bus $k$, during hour $t$, is $g_{k}(t)$. The cost of generation is given by $C_{k}\left[g_{k}(t)\right]$ including start-up and other costs. Power distribution and consumption agents operate at load substation busses. $\mathrm{D}$ and $\mathrm{C}$ agents predict power demand at bus $k$ during hour $t$ as $d_{k}(t)$. The power demand is assumed inelastic, i.e. 
fixed for any price signal. Renewable generation is becoming widely used through small-scale technologies that produce low-cost, reliable, and clean electricity close to customers. The system total demand, during hour $\mathrm{t}$, is computed as the sum of $d_{k}(t)$ and is given by $D(t)$. Agents are assumed to implement dynamic scheduling of dispatchable generation, demand-side management techniques, consolidation of load balances for separate power zones represented at bus $k$, and flexible energy storage. The transmission agent coordinates the physical operation and controls the entire system in real-time in coordination with SCADA control center. It maintains [1]:

- generation-load balance at time $t, \sum_{k, t} g_{k}(t)=D(t)$;

- enough spinning reserve $r$ at bus $k$, time $t, \sum_{i, t} g_{i}(t)-g_{k}(t) \leq r_{k}(t)$;

- min-max available bus generation, at time $t g_{k, \min } \leq g_{k}(t) \leq g_{k, \max }$;

- flow limits on transmission lines: $\left|z_{l}(t)\right|=z_{l, \max }$.

\subsection{Agent Classes}

The generation, distribution, customer, and transmission agents are setup with adaptable properties [18]. As shown in Figure 3, each agent extends from the abstract class MonitorableAgent and integrates with variety of other classes including MonitoringConstants and SystemConstants interfaces. The systemConstants Interface include: sniffer_agent, system_runner_agent, distribution_agent, transmission_agent, generation_agent, and customer_agent. T Agent represents

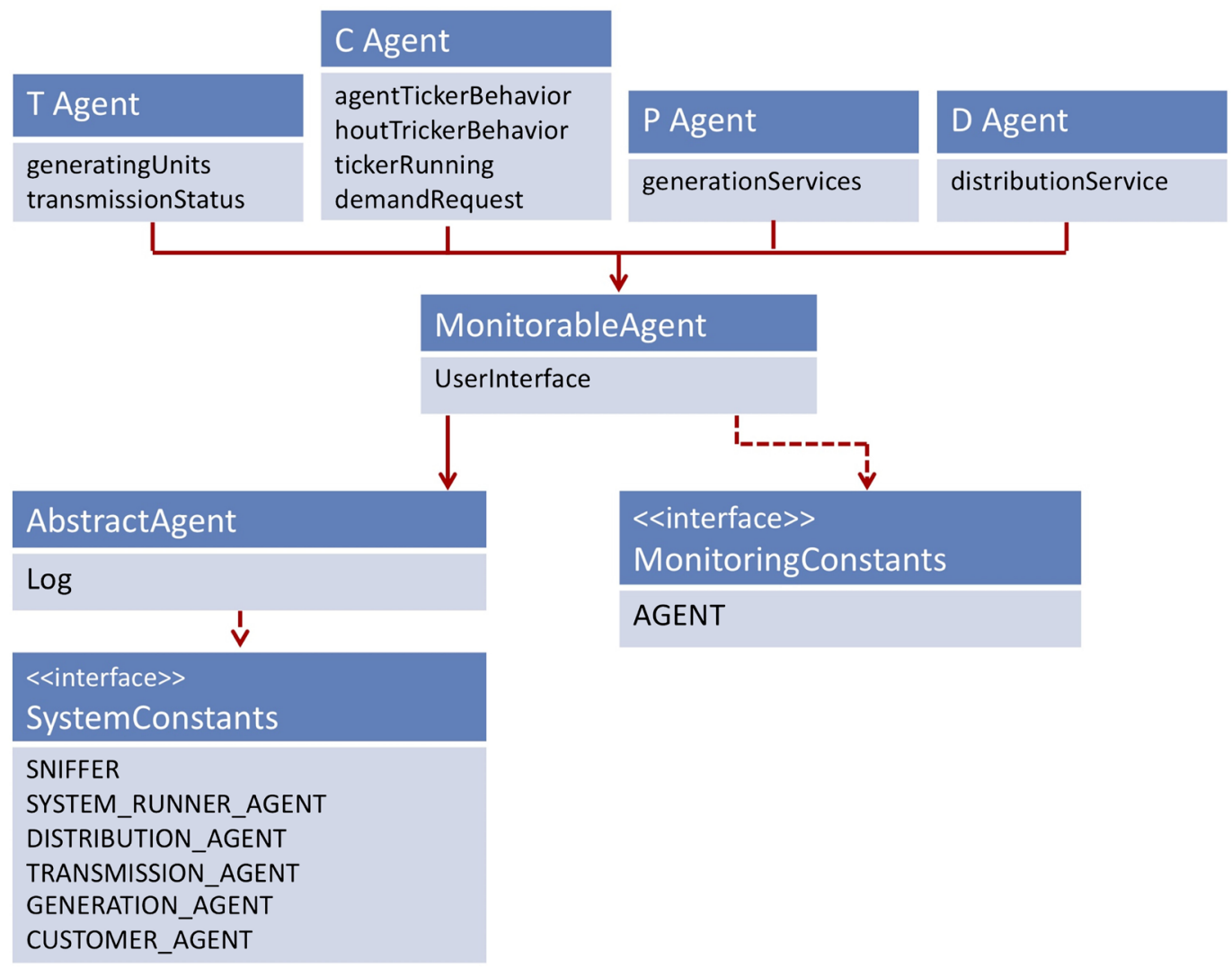

Figure 3. Agent class diagram. 
behaviors that interact with generation and load balance and maintain real-time power flow. C Agent has agentTickerBehaviour and hourTickerBehaviour fields that store custom agent behavioral characteristics. Fields tickerRunning and demandRequest are used to track current status and important power demand updates. $\mathrm{P}$ Agent has a generation services field that includes information for each generation unit on power production and spinning reserves for different time intervals. D Agent has a distribution services field that includes information on power demand and load profiles for different time intervals and for each substation/load bus. The systemRunnerAgent represents an important class for all agents. It facilitates a setup and registration to defining agent description and startup phase.

\subsection{Agent Communication}

Agents interact and communicate asynchronously with their peers. Each agent has a mailbox which notifies the agent when a new message arrives. Figure 4 shows the JADE messaging system. Inter-agent communication is implemented as an object derived from the agent transparency class. It selects the best route and finds a compromise on message semantics and format to make their communications easier. All messages adhere to ACL standards, namely:

Message source and communicative intention ("performativity").

Message content, language, conversation ID, and ontology.

\section{Illustrative Example}

The Jordan 51-bus system was used for simulation. $230 \mathrm{MW}$ of the $4050 \mathrm{MW}$ total generation capacity represented renewable energy [18] [19] [20] [21]. The growing power demand, high costs of imported fuel, lack of water cooling, and frequent cascading blackouts represent inevitable challenges to the Jordanian electric power system. The economic analyses were demonstrated on the use of smart grid technologies with 2016 generation-load profiles. In order the validate how agents respond to variations in power costs, $\pm 20 \%$ of liquefied natural gas (LNG) prices for sensitivity analysis were used. The analyses provide insights on the robustness of agents for smart power grids.

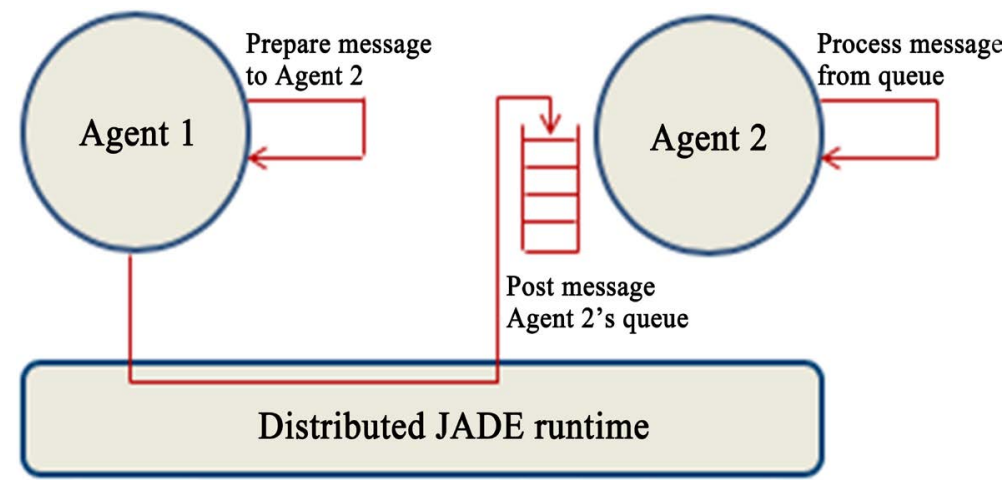

Figure 4. Message transmission on JADE platform. 


\subsection{Operating Costs}

Operation planning requires day-to-day load profiles for unit commitment and power dispatch of available resources provided that security, stability, and reliability constraints are met. Generation changes on hourly basis and schedules of unit commitment and power dispatch are computed based on estimated load profiles and operational constraints. The presence of a significant amount of non-dispatchable and non-predictable renewable generation implies an update of the system operational requirements, mainly in regard to operating reserves. To assess the impact of MAS technology on the production costs of electricity, a day-ahead simulation has been used.

Renewable energy resources produce electricity to compensate for energy that could otherwise be produced by conventional power plants. Comparing energy production with and without renewable resources, it is possible to evaluate in quantitative terms the differences in electricity production costs, marginal costs, and displaced energy resources for different technologies. The increased penetration of renewable energies in the Jordanian generation mix contributes to economic costs and savings. These costs and savings are calculated on the basis of different scenarios of MAS simulations.

Operational costs of generation units are assumed quadratic and a centralized unit commitment and power dispatch mechanisms are implemented by the $\mathrm{T}$ Agent. The simulation results give the optimum schedule of resources with minimum cost of power generation. An equivalent network model is used with interconnected zones transferring power at maximum capacity. Interconnected neighboring electric systems are modelled with equivalent generating units. Negative production means that energy has been exported while positive production means that energy has been imported with neighboring grids. Thermal generating units are characterized by their company owner, zone, min-max capacity, fuel mix and prices, quadratic cost function, average forced outage rate, and start-up/shut-down constraints.

\subsection{Simulation Results}

MAS is implemented with agents representing power generation, distribution, and transmission. The set of power generation is optimally coordinated with renewable resources in a one-year time frame and on an hourly base discretization. This gives a total of 8760 hours that are considered for analysis. A day-ahead hourly energy system is simulated while considering system-wide marginal pricing and congestion management. The base case is investigated with different scenarios for year 2016. The average hourly prices of power are shown in Figure 5 for both agent-based and no-agent power systems for a nominal liquified natural gas (LNG) prices for a summer day. Hourly prices are higher for real operation during peak hours. Sensitivity analysis is performed for LNG with $\pm 20 \%$ of fuel prices. Figure 6 and Figure 7 give comparison of average hourly prices of power for both agent-based and no-agent power systems for LNG $\pm 20 \%$. The 


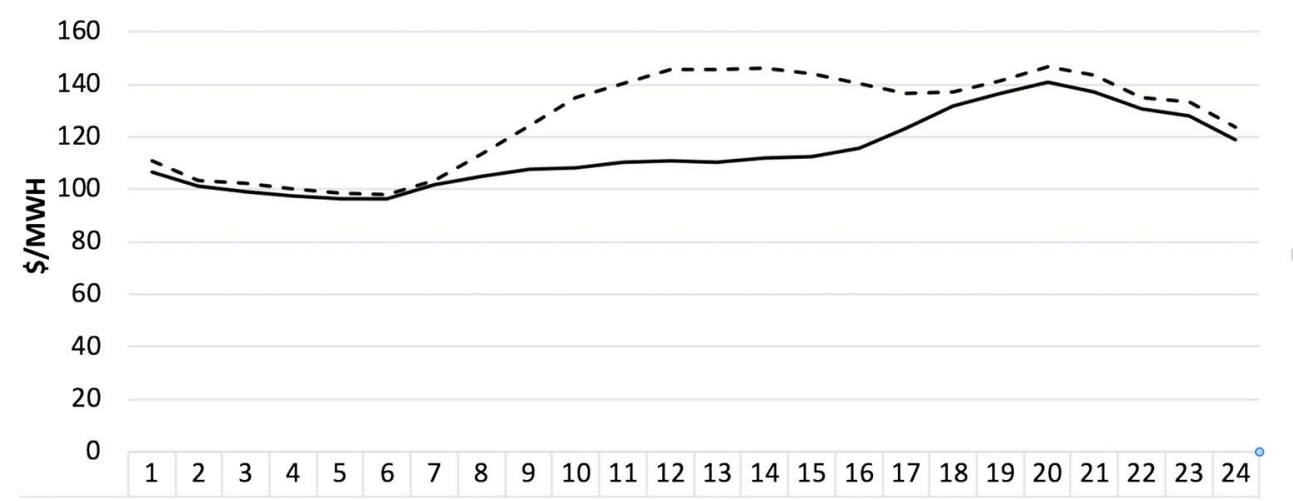

Figure 5. Average hourly prices for agent-based and no-agent LNG power system.

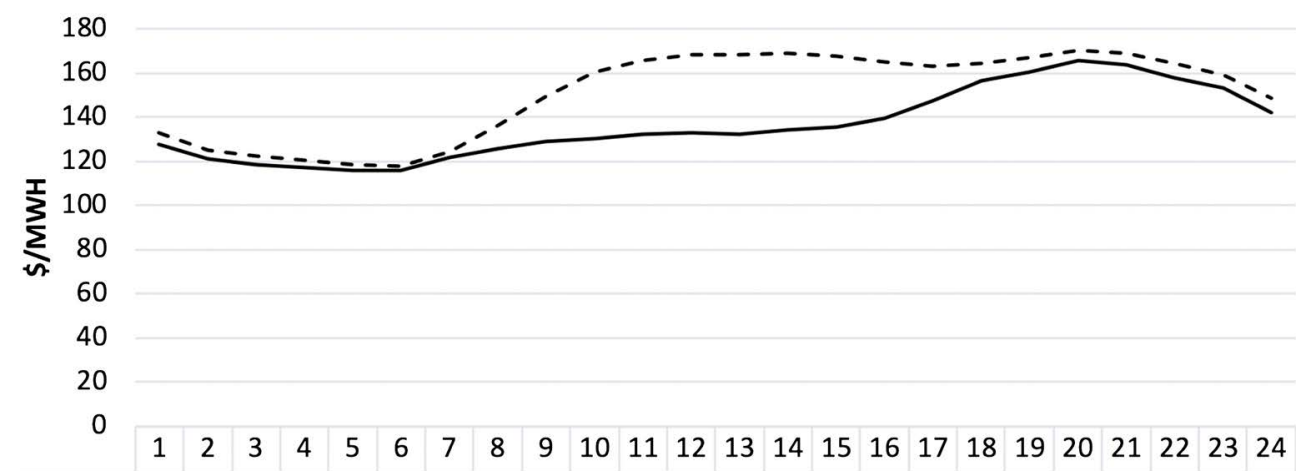

Figure 6. Average hourly prices for agent-based and no-agent LNG $+20 \%$ power system.

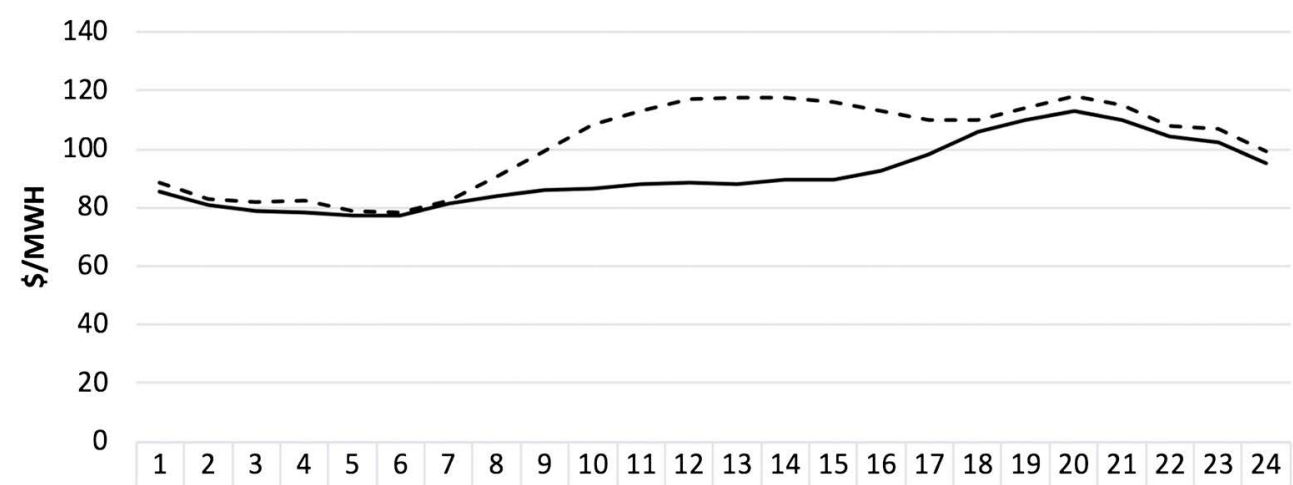

Figure 7. Average hourly prices for agent-based and no-agent LNG $-20 \%$ power system.

one-day analysis shows that energy prices are higher during peak hours with no MAS technologies integrated to accommodate real-time control of renewable energies (shown as dotted lines in the graph).

The simulation has also been run for a full year 2016. Table 1 shows a summary of fuel costs and average energy prices for LNG and LNG $\pm 20 \%$ operation scenarios. The total LNG fuel cost is \$2056.4 million with agent-based simulation compared to $\$ 2333.1$ million with real operation. This gives an average Megawatt hour price of $\$ 120.8$ with agent-based simulation compared to $\$ 122.5$ with real operation.

The total LNG $+20 \%$ fuel cost is $\$ 2456.6$ million with agent-based simulation 
Table 1. Summary of fuel costs and average prices for different types of operation scenarios.

\begin{tabular}{cccc}
\hline Scenarios & Type of Operation & Fuel Cost (M\$) & Average Price (\$/MWh) \\
\hline \multirow{2}{*}{ LNG Base case } & MAS based operation & 2056.4 & 120.8 \\
& Real operation & 2333.1 & 122.5 \\
\multirow{2}{*}{ LNG $+20 \%$} & MAS based operation & 2456.6 & 143.6 \\
& Real operation & 2748.3 & 144.6 \\
LNG $-20 \%$ & MAS based operation & 1645.1 & 96.7 \\
\hline
\end{tabular}

compared to $\$ 2748.3$ million with real operation. The average Megawatt hour price increased from $\$ 120.8$ to $\$ 143.6$ with agent-based simulation compared to an increase from $\$ 122.5$ to $\$ 144.6$ with real operation. On the other hand, the total LNG $-20 \%$ fuel cost is $\$ 1645.1$ million with agent-based simulation compared to $\$ 1866.5$ million with real operation. The resulting average Megawatt hour price decreased from $\$ 120.8$ to $\$ 96.7$ with agent-based simulation compared to a decrease from $\$ 122.5$ to $\$ 101.2$ with real operation. These variations are mainly driven by the fact that agents shift power generation to renewable power plants to produce maximum power at peak hours. As a result, there is a positive economic impact in both cases, due to the fact that agents coordinate to better displace expensive thermal generation with renewable generation. It is evident that renewable resources compensate for power at peak times and provide economic benefits and savings.

In economic terms, an average saving of $0.0079 \%$ in energy costs is made when using agent technologies. Assuming an average daily generation capacity of $3500 \mathrm{MW}$, then the amount of yearly savings will be $\$ 35$ million. It is clear that MAS technologies reduce fuel costs and energy prices as they drive renewable resources to compensate for power at peak time and consequently, provide economic benefits and savings. The results of this research are consistent with the results of MAS research in power grid systems [2] [3] [19] in support the use of agents as viable tools for power system management and energy efficiency.

\section{Conclusion}

A conceptual agent design has been presented for a smart grid system using agent technologies. The agents act as smart self-organizing elements, which inherent a distributed nature of adaptive interacting systems. It has been shown that agents are important technologies for setting up smart grids. The economic impact of agent integration with renewable resources is noticeable. The economic analyses are performed for the year 2016, both for MAS integration and real operation with respect to price of fuel gas and operating reserves. A day-ahead simulation has been used in order to simulate hourly generation costs. The results of the simulation show an average saving of $0.0079 \%$ in energy 
costs when using agent technologies. Assuming an average daily generation capacity of $3500 \mathrm{MW}$, then the amount of yearly savings will be $\$ 35$ million. It should be noted that MAS technologies may be explored for different grid systems while taking into account the intermittent nature of renewable resources and unpredictable weather conditions. It is anticipated that MAS technologies can be useful for further research and would support the development of smart grid systems in a larger context.

\section{Conflicts of Interest}

The authors declare no conflicts of interest regarding the publication of this paper.

\section{References}

[1] Al-Agtash, S. (2012) Electricity Agents in Smart Grid Markets. Computers in Industry, 64, 235-241. https://doi.org/10.1016/j.compind.2012.10.009

[2] Khazaei, J. and Nguyen, D. (2019) Multi-Agent Consensus Design for Heterogeneous Energy Storage Devices with Droop Control in Smart Grids. IEEE Transactions on Smart Grid, 10, 1395-1404. https://doi.org/10.1109/TSG.2017.2765241

[3] Lau, J., Huang, G., Mak, K. and Liang, L. (2006) Agent-Based Modeling of Supply Chains for Distributed Scheduling. IEEE Transactions on Systems, Man, and $C y$ bernetics, 36, 847-861. https://doi.org/10.1109/TSMCA.2005.854231

[4] Bose, A. (2010) Smart Transmission Grid Applications and Their Supporting Infrastructure. IEEE Transactions on Smart Grid, 1, 11-19. https://doi.org/10.1109/TSG.2010.2044899

[5] Bintoudi, A., Zyglakis, L., Tsolakis, A., Ioannidis, D., Hadjidemetriou, L., Zacharia, L., Mutlaq, N., Hashem, M., Al-Agtash, S., Kyriakides, E., Demoulias, C. and Tzovaras, C. (2020) Hybrid Multi-Agent-Based Adaptive Control Scheme for AC Microgrids with Increased Fault-Tolerance Needs. IET Renewable Power Generation, 14, 1-6. https://doi.org/10.1049/iet-rpg.2019.0468

[6] Lopez-Rodriguez, I., Hernandez-Tejera, M. and Lopez, A. (2016) Methods for the Management of Distributed Electricity Networks Using Software Agents and Market Mechanisms: A Survey. Electric Power Systems Research, 136, 362-369. https://doi.org/10.1016/j.epsr.2016.03.016

[7] Molderink, A., Bakker, V., Bosman, M., Hurink, J. and Smit (2010) Management and Control of Domestic Smart Grid Technology. IEEE Transactions on Smart Grid, 1, 109-119. https://doi.org/10.1109/TSG.2010.2055904

[8] Rohbogner, G., Fey, S., Hahnel, U., Benoit, P. and Wille-Haussmann, B. (2012) What the Term Agent Stands for in the Smart Grid Definition of Agents and Multi-Agent Systems from an Engineer's Perspective. Proceedings of the 2012 Federated Conference on Computer Science and Information Systems, Wroclaw, 9-12 September 2012, 1301-1305.

[9] National Institute of Standards and Technology (2010) NIST Framework and Roadmap for Smart Grid Interoperability Standards Release 1.0.

[10] Spataru, C. and Barrett, M. (2013) Smart Consumers, Smart Controls, Smart Grid. In: Hakansson, A., Höjer, M., Howlett, R. and Jain, L., Eds., Sustainability in Energy and Buildings, Smart Innovation, Systems and Technologies, Vol. 22, Springer, Berlin, Heidelberg, 381-389. https://doi.org/10.1007/978-3-642-36645-1_36 
[11] Li, F., Qiao, W., Sun, H., Wan, H., Wang, J., Xia, Y., Xu, Z. and Zhang, P. (2010) Smart Transmission Grid: Vision and Framework. IEEE Transactions on Smart Grid, 1, 168-177. https://doi.org/10.1109/TSG.2010.2053726

[12] Shawon, M., Muyeen, S., Ghosh, A., Islam, S. and Baptista, M. (2019) Multi-Agent Systems in ICT Enabled Smart Grid: A Status Update on Technology Framework and Applications. IEEE Access, 7, 97959-97973. https://doi.org/10.1109/ACCESS.2019.2929577

[13] Rahman, S., Pipattanasomporn, M. and Teklu, Y. (2007) Intelligent Distributed Autonomous Power Systems. Proceedings of 2007 IEEE Power Engineering Society General Meeting, Tampa, 24-28 June 2007, 1-8. https://doi.org/10.1109/PES.2007.386043

[14] Bellifemine, F., Caire, G. and Greenwood, D. (2008) Developing Multi-Agent Systems with JADE. John Wiley \& Sons, Hoboken.

https://doi.org/10.1002/9780470058411

[15] Wooldridge, W. (2009) An Introduction to Multiagent Systems. John Wiley \& Sons, Hoboken.

[16] Jain, M., Gupta, S., Masand, D., Agnihotri, G. and Jain, S. (2016) Real-Time Implementation of Islanded Microgrid for Remote Areas. Journal of Control Science and Engineering, 2016, Article ID: 5710950. https://doi.org/10.1155/2016/5710950

[17] Brown, R. (2008) Impact of Smart Grid on Distribution System Design. Proceedings of 2008 IEEE Power and Energy Society General Meeting-Conversion and Delivery of Electrical Energy in the 21 st Century, Pittsburgh, 20-24 July 2008, 1-4. https://doi.org/10.1109/PES.2008.4596843

[18] AbdelHafez, H. (2017) Multi Agent Systems for Smart Power Grids. Master Thesis, German Jordanian University, Amman.

[19] Elsied, M., Oukaour, A., Gualous, A. and Ottavio, B. (2016) Optimal Economic and Environment Operation of Micro-Grid Power Systems. Energy Conversion and Management, 122, 182-194. https://doi.org/10.1016/j.enconman.2016.05.074

[20] Alzyoud, F., Alsharman, N. and Almofleh, A. (2019) Best Practice Fundamentals in Smart Grids For a Modern Energy System Development in Jordan. Proceedings of the 9 th International Conference on Advances in Computing, Communication and Information Technology, Rome, 7-8 December 2019, 80-86.

[21] Jordan National Electric Power Company NEPCO (2015) The 2015 Annual Report. http://www.nepco.com.jo/store/docs/web/2015_en.pdf 